\title{
Earth's field effect on magnetic performance of horizontally and vertically polarizing undulators
}

\author{
N. O. Strelnikov ${ }^{1,2, *}$ and I. B. Vasserman ${ }^{1, \dagger}$ \\ ${ }^{1}$ Advanced Photon Source, Argonne National Laboratory, Argonne, Illinois 60439, USA \\ ${ }^{2}$ Budker Institute of Nuclear Physics, Novosibirsk 630090, Russia
}

(Received 9 December 2013; published 11 June 2014)

\begin{abstract}
Effects of the interaction of the Earth's magnetic field with different types of insertion devices (IDs) were studied at the Advanced Photon Source (APS) at Argonne National Laboratory (Argonne) according to the improvement of high-precision field measurements and field correction techniques that should meet the tight requirements for next-generation x-ray sources. The magnetic measurements of two Advanced Photon Source hybrid-type IDs with periods of 33 and $27 \mathrm{~mm}$ have been performed. The impact of different design elements was considered. It was demonstrated that the specified limits for IDs can be exceeded if the effect of the Earth's field is not taken into account for various orientations of the IDs in the storage ring. Numerical simulations were done to better interpret experimental results for both devices and to estimate the effect for novel ID with the horizontal main field, which is now under development.
\end{abstract}

DOI: 10.1103/PhysRevSTAB.17.062401

PACS numbers: 07.55.Db, 07.85.Qe, 41.60.Ap

\section{INTRODUCTION}

The magnetic performance of undulators at third-generation synchrotron radiation (SR) sources has been studied and improved significantly over the past decade [1-3]. Undulators have delivered predicted bright radiation beams and at the same time have met requirements to be transparent to the electron beam in storage rings. These requirements directly depend on the emittance of the storage ring [4], and with new designs of diffraction-limited x-ray SR sources it is important to identify and mitigate all possible e-beam perturbations caused by insertion devices (IDs). One such perturbation is related to the fact that IDs are typically prepared and tuned in a magnetic environment that could differ from the operational one. Specifically, the orientation of the ID's magnetic structure and its frame with respect to the vector of the Earth's magnetic field at the location of ID magnetic measurement bench in Advanced Photon Source (APS) Magnetic Measurement Facility (MMF) differs from those at many straight sections of the Argonne National Laboratory's (Argonne's) APS storage ring. Such variations lead to differences in the penetration of the Earth's magnetic field in the ID gap space, as well as differences in interactions of that field with magnetically susceptible elements of ID mechanical structures. An accurate assessment of the influence the Earth's magnetic field has on values and variations of an ID's field

\footnotetext{
*niks.str@gmail.com

†vasserman@aps.anl.gov
}

Published by the American Physical Society under the terms of the Creative Commons Attribution 3.0 License. Further distribution of this work must maintain attribution to the author $(s)$ and the published article's title, journal citation, and DOI. integrals for different magnetic gaps is important for any ID installed in the storage ring. However, this is even more significant for future SR diffraction-limited sources. Such sources with ultralow emittance will utilize on-axis e-beam injection and, as a result, new types of IDs with horizontal main magnetic fields could become as common as currently standard IDs with main vertical fields. Therefore, the influence of the Earth's magnetic field on the magnetic performance of different types of IDs in different orientations has to be evaluated. Some consideration of this task has already been done $[5,6]$. This paper presents experimental and modeling results concerning the effects of the interaction of Earth's magnetic field with different types of IDs. For experimental studies, two APS hybrid-type IDs with periods of 33 and $27 \mathrm{~mm}$ were used.

\section{MEASUREMENTS OF THE EFFECTS OF THE EARTH'S FIELD PROPAGATION IN THE ID GAP}

Although the absolute value of the Earth's magnetic field is much smaller than the main field inside the ID's gap, the Earth's field contribution to integrals and multipole components, both vertical and horizontal, on the ID length could be quite significant and even exceed specification requirements. The requirements for the APS insertion devices were set by the APS team. These requirements result from the need to provide beam stability at $10 \%$ of particle beam emittance [7]. Usually, tuning of the devices includes the Earth field at the APS Magnetic Measurement Facility (MMF), and the propagation of the field and its effect on the field integrals and multipole components are assumed to remain the same during operation. Recently it was revealed that Earth field propagation is affected rather strongly by the orientation of the device, and that this effect can cause distortions when tuning the device according to 
specifications in the MMF. An improved storage ring feedback system currently allows these errors to be corrected; however, future APS upgrades will assume horizontal emittance that decreases by almost 2 orders of magnitude, so the requirements for beam stability will become much tighter.

Appendix A contains a description of the ID's field integrals and their effect on the charged particle beam passing the ID. The vertical component of Earth's magnetic field introduces an orientation-independent addition to the field integrals for both types of IDs, with a vertical or horizontal main magnetic field. The influence of the horizontal component of Earth's magnetic field on the ID's horizontal field (transverse to the ID's longitudinal axis) depends on the orientation of the ID in the storage ring tunnel. This is the case for both types of ID with vertical and horizontal main magnetic field. As a result, the values of the transverse horizontal field integrals of such IDs in the storage ring tunnel could differ from those measured at the Magnet Measurement Facility (MMF) of the APS. The input of the Earth's horizontal field component on the ID's transverse horizontal field integral also depends on the ID's gap (Appendix B), as well as the material and geometry of the ID support and drive frame. All these gap- and positiondependent effects in addition to the potential influence of the ID's frame on the ID's field integrals can and should be studied experimentally by using different types of APS IDs and utilizing precise measurement techniques at the APS MMF.

Only one of the two components of the horizontal part of the Earth's magnetic field can contribute to the ID field integral: the component perpendicular to the longitudinal axis of the ID. Therefore, only that component will be considered in further analysis. A simple way to measure the field integral of the transverse horizontal component of the Earth's field is to measure ID field integrals for two ID orientations that differ by $180^{\circ}$ rotation around the vertical axis (the $y$ axis). In this case,

$J_{1 x}(0)=J_{1 x U}+J_{1 x E}$-first field integral of the horizontal field, original orientation,

$J_{1 x}(180)=-J_{1 x U}+J_{1 x E}$-first field integral, rotated $180^{\circ}$ around the $y$ axis.

Here, $J_{1 x U}$ is the undulator magnetic structure contribution, and $J_{1 x E}$ is the Earth's field contribution. It follows from these equations that $J_{1 x E}=\left[J_{1 x}\left(0^{\circ}\right)+J_{1 x}\left(180^{\circ}\right)\right] / 2$.

Two APS IDs, APS27 \#5s and UNA33 \#6, which have 27-mm and 33- $\mathrm{mm}$ periods, respectively, have been chosen for the measurements performed at the APS MMF. The frame of the APS27 \#5s is made of steel, which could be magnetized; the frame of the UNA33 \#6 is practically nonmagnetic. Therefore, comparing measurements for these two devices should reveal the effect of the ID frame on the field integrals. In addition, preliminary measurements without IDs have been performed to define the Earth's field value at the measurement area.
A long, stretched, rotated coil was used for the measurements. The measurements in the case of no ID present with a 4.2-m-long rotating coil give a value of $J_{1 x}=$ $-63 \mathrm{G}-\mathrm{cm}$ for the transverse horizontal field and $J_{1 y}=$ $-167 \mathrm{G}-\mathrm{cm}$ for the vertical. That corresponds to -0.15 gauss average horizontal field and -0.4 gauss average vertical field in the vicinity of the magnet measurement bench. The distribution of the Earth's field in this area is close to constant; therefore the approximation in the data processing of the average field is acceptable. The measurements of $J_{1 x E}$ for both devices are shown in Fig. 1. While measurements are being made, IDs are oriented so that $J_{1 x E}$ is at its maximum.

It follows from Fig. 1 that the maximum field integral variations of $J_{1 x E}$ are $28 \mathrm{G}-\mathrm{cm}$ and $39.5 \mathrm{G}-\mathrm{cm}$ for UNA33 \#6 and APS27 \#5s, respectively. Since the tuning process stops when field integrals and field integral gap dependence (integrals variation with gap changing) satisfy the requirements, the integrals and gap dependence are not tuned to zero values. If the devices have the same orientation in the storage ring tunnel as in the MMF, their field integral gap dependencies would be equal to those obtained during the tuning at the MMF. In the worst case of the ID location, integral variations for the two IDs shown in Fig. 1 (28 G-cm for UNA33 \#6 and 39.5 G-cm for APS27 \#5s) should be taken into account in order to keep the variation of the first horizontal field integral as a function of the ID gap within the $50 \mathrm{G}-\mathrm{cm}$ specification. This means that each ID cannot be tuned universally, and tuning should take into account its orientation in the ring.

As shown in Fig. 1, the horizontal component of the Earth's field at gaps $30-150 \mathrm{~mm}$ is almost the same for both devices; therefore, the effect of the frame material on the transverse horizontal Earth's field propagation into the ID gap is very small. The difference between curves at gaps smaller than $30 \mathrm{~mm}$ in Fig. 1 cannot be explained by different frame material; it requires further investigation. Shielding of the horizontal Earth's field component by poles can be seen in this figure as well. It occurs at all gaps,

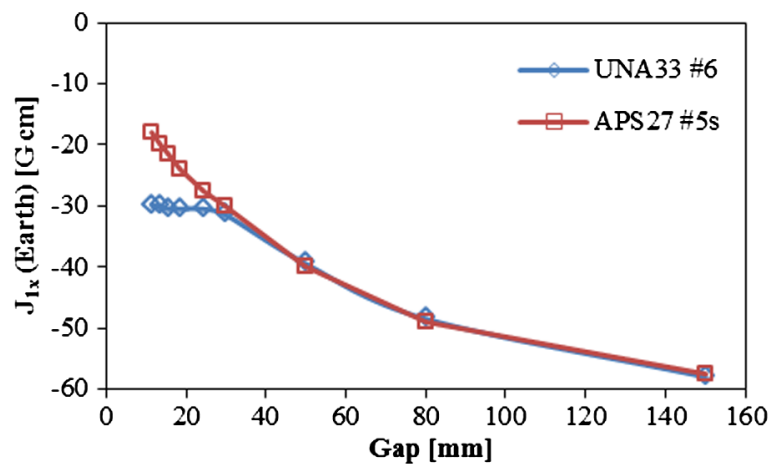

FIG. 1. Effects of the propagation of the transverse horizontal Earth's field component in the UNA33 \#6 and in the APS27 \#5s. First field integral gap dependence is presented. 
even in the biggest gap: the field integral measured in the $150-\mathrm{mm}$ gap is $-57.5 \mathrm{G}-\mathrm{cm}$, and the same integral measured without devices is $-635 \mathrm{G}-\mathrm{cm}$.

\section{VERTICAL EARTH'S FIELD PROPAGATION}

As mentioned above, the propagation of the vertical Earth's field component should not depend on device orientation if its magnetic structure is ideal, and if there are no extra magnetic materials close to the magnetic structure. However, measurements of the vertical field integrals were different for the original orientation of the devices in the MMF compared to those rotated $180^{\circ}$ (Fig. 2).

This can probably be explained by magnetic structure asymmetry, which takes place for both devices (but on different levels). In this case, the expression "vertical Earth's field propagation" refers to the influence of the horizontal Earth's field component on the vertical field integral.

At open gaps, UNA33 \#6 has zero difference from the first field integrals because asymmetry of the magnetic structure of this device becomes negligible. The case with APS27 \#5s has to be considered more closely. Strong multipole components were measured for this device, and were tuned for nonrotated orientation of the device. This explains the nonzero $J_{1 y}$ difference of the ASP27 device, both rotated and in the original orientation, in the open gap (80-150 mm). As will be shown in Sec. IV B, a magnetically susceptible frame (APS27 \#5s has a frame made of magnetically susceptible steel) does not introduce visible asymmetry for vertical external field propagation (see Fig. 8), but it shields the vertical external field by decreasing the total flux passing through poles (the magnetic frame shielding will be considered further). The difference between the curves in Fig. 2 could be explained by these differences between these two devices.

In the case of magnetic structure asymmetry in the $x$ direction (because of design features), significant multipoles can be generated by this asymmetry; these are dependent on the device's orientation with respect to the Earth's magnetic field vector. To verify this, the multipoles of UNA33 \#6 were measured for both a nonrotated and a

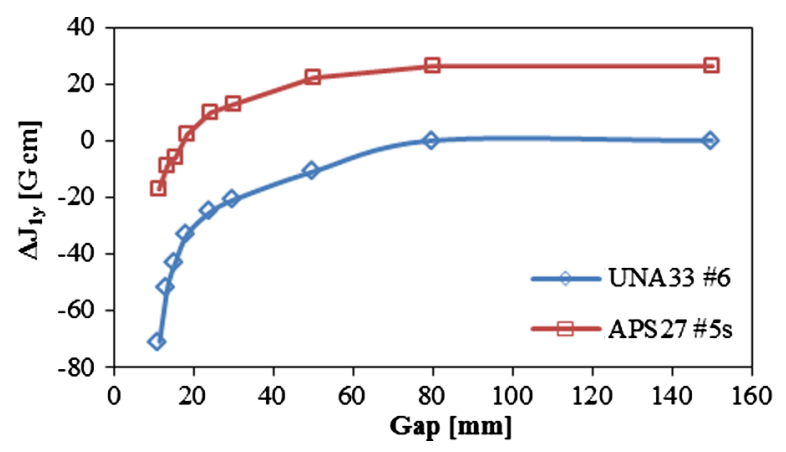

FIG. 2. The difference in first field integrals of two IDs, original and $180^{\circ}$ rotated orientations; one ID has a magnetically susceptible frame and the other is nonmagnetic.

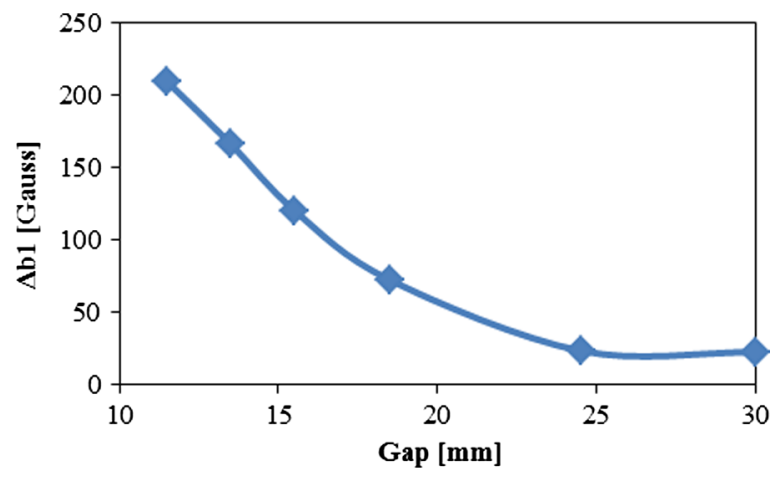

FIG. 3. Difference of the normal integrated quadrupole component $\left[\mathrm{b} 1\left(180^{\circ}\right)-\mathrm{b} 1\left(0^{\circ}\right)\right]$ between the rotated and nonrotated ID UNA33 \#6.

rotated device. The difference between the normal integrated quadrupole component of a polynomial fitting (b1) for two orientations of the device is shown in Fig. 3. The integrated quadrupole component for the nonrotated device was compensated during the tuning process and had b1 $<50$ gauss for all gaps.

A strong difference (shown in Fig. 3) for the b1 component proves the device asymmetry, which should be taken into account during tuning. It is worth mentioning that this device asymmetry is much smaller than that of APS27 \#5s, according to results of measurements taken in the MMF. For example, the skew quadrupole component at gap $30 \mathrm{~mm}$ is 38 gauss for UNA33 \#6 compared to 131 gauss for APS27 \#5s.

\section{NUMERICAL SIMULATION}

Numerical simulations were performed in order to understand the experimental results better and to investigate field effects caused by the magnetic frame. During simulations, the geometries of both devices (APS27 \#5s and UNA33 \#6) were used. The calculations were done by means of the RADIA [8,9] and OPERA [10] codes. The Earth's magnetic field, as measured without a device, was used for simulations ( $B_{x}=-0.15$ gauss and $B_{y}=-0.4$ gauss).

\section{A. Calculations with and without magnets}

Since magnetic measurements are sensitive enough to detect small effects of the Earth's field and ID magnetic environment, the modeling has to include all possible details of real ID geometry, such as the actual ID frame configuration, and total magnetic length. Calculations that include all these details are very time consuming; therefore magnets have been removed from the simulation model. It is assumed that the poles are not saturated and that magnets do not affect the Earth's field penetration into the poles. The calculation of a 1.5-period model with and without magnets was performed by the OPERA code to prove this assumption. The calculation was done with maximum achievable accuracy on available machine resources. 

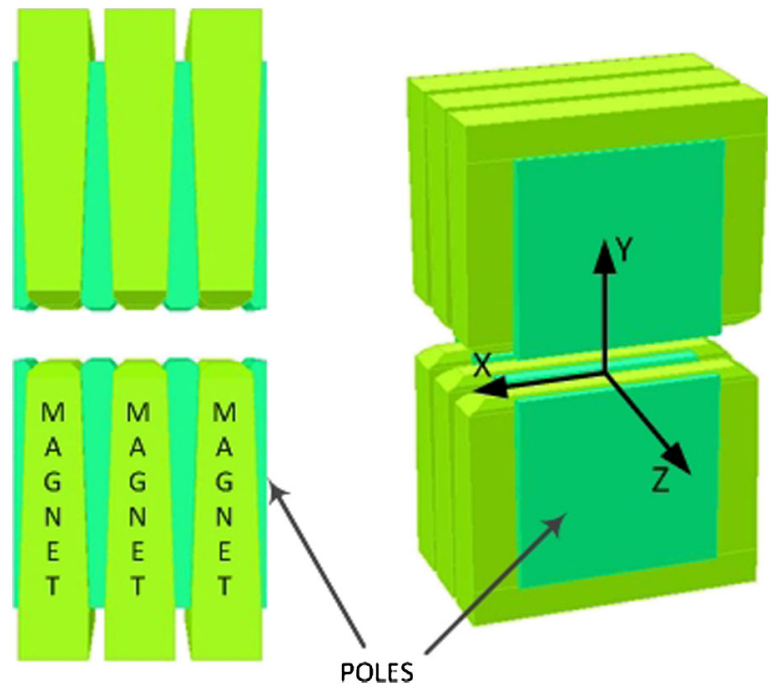

FIG. 4. The simulation model (with magnets) for the UNA33 \#6 geometry.

Symmetric boundary conditions were applied with the normal-to-transverse vertical plane ( $x y$ ) field component equal to zero. The simulation model "with magnets" for the UNA33 \#6 geometry is shown in Fig. 4. The calculation was performed for the minimum gap used during the ID operation $(10.5 \mathrm{~mm})$ to obtain maximum field values, which provides the maximum effect to be checked. The $B_{y}=-0.4, B_{x}=-0.15$ external field was set. The calculation data obtained for the field integral distributions along the $x$ axis are presented in Fig. 5.

There are nonsystematic errors introduced by adding magnets into the calculation. As shown, the average integral value for a simulation with magnets is the same as for a simulation without magnets. This makes the above assumption correct and allows calculations without magnets to be carried out.

\section{B. Full model simulation}

The calculations for APS27 \#5s were done using the RADIA and OPERA codes to ensure accuracy in the obtained

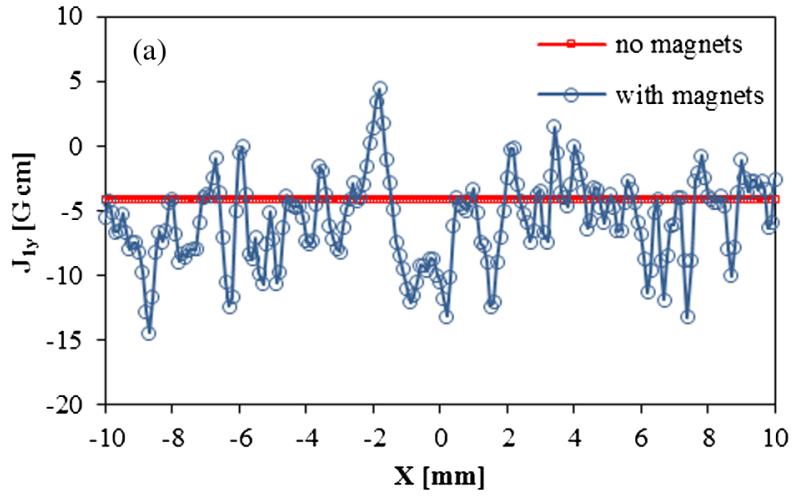

results. The simulations were conducted for a set of poles only, both with and without a frame taken into account. To check the experiment results, two values of the external field $\left(B_{y}=-0.4, B_{x}=-0.15\right.$ and $\left.B_{y}=-0.4, B_{x}=0.15\right)$ were set during the calculations with a frame. The simulation model of the APS27 \#5s with a frame is shown in Fig. 6. Because of the simulation model symmetry, the calculations for UNA33 \#6 with a nonmagnetic frame were done with RADIA only, for one external field value $\left(B_{y}=-0.4, B_{x}=-0.15\right)$.

\section{Horizontal field propagation}

The gap dependence of the transverse horizontal field integrals $\left(J_{1 x}\right)$ can be observed in Fig. 7, as calculated in the median plane of APS27 \#5s with the RADIA and OPERA codes. There are curves corresponding to simulations both with and without a frame (external field: $B_{y}=-0.4$, $B_{x}=-0.15$ gauss). In addition, in Fig. 8 there is a comparison of the propagation of differently directed external fields $\left(B_{y}=-0.4, B_{x}=-0.15\right.$ and $B_{y}=-0.4$, $B_{x}=0.15$ gauss) that correspond to the cases of a nonrotated device and one rotated by $180^{\circ}$. All integrals for both devices were calculated on a 4200-mm length, which corresponds to the long, rotated coil length.

The discrepancies between the RADIA and OPERA simulations without frame are within the calculation error. The differences with frame can be explained by the finite surrounding area in the OPERA simulation model, which limits full flux through the frame and poles. It follows from Figs. 8 and 9 that the frame effect on the transverse horizontal Earth field propagation is very small, and the frame does not introduce any asymmetry for external transverse horizontal field propagation.

\section{Vertical field propagation}

The frame effect on the vertical Earth field propagation was also checked. No asymmetry was detected for external vertical field propagation (Fig. 9).

The difference between calculations with a frame and without a frame is rather significant in the case of the

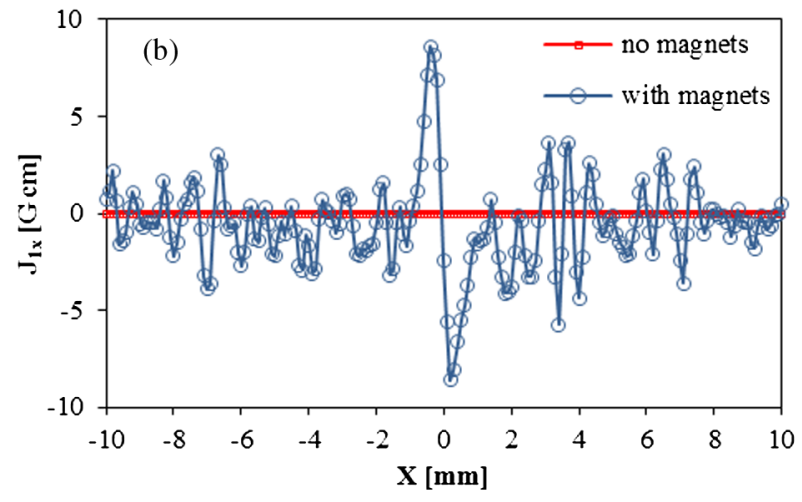

FIG. 5. $J_{1 y}$ vs $x$ (a) and $J_{1 x}$ vs $x$ (b) calculations with and without magnets for UNA33 \#6. 


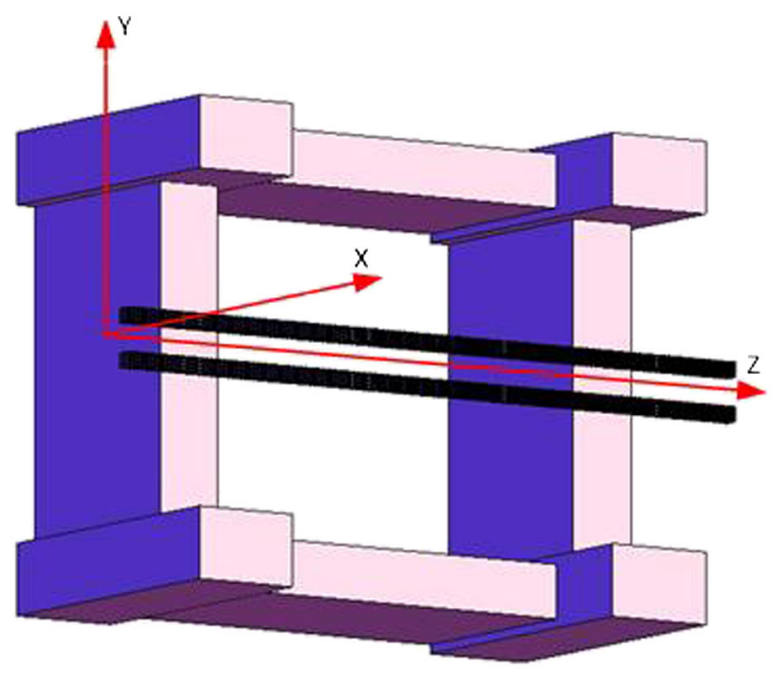

FIG. 6. The simulation model of the APS27 \#5s with a frame.

Earth's vertical field (Fig. 10). The magnetic frame attracts an external field, thus decreasing the flux going through the ID's poles. In other words, it shields the vertical field.

The variation of the first vertical field integral obtained in the simulation vs gap change for APS27 \#5s with the frame is $24 \mathrm{G}-\mathrm{cm}$; without a frame, it is $82 \mathrm{G}-\mathrm{cm}$ (Fig. 10). The last value is very close to the APS ID's specifications (100 G-cm for vertical field). The result obtained from similar calculations for UNA33 \#6 with a nonmagnetic frame is $96 \mathrm{G}-\mathrm{cm}$.

Unlike the horizontal component of the Earth's field, its vertical field is enhanced by the poles of the ID and decreasing the gap increases enhancement (Fig. 11).

The enhancement of the external vertical field by the ID's poles is twice stronger for the nonmagnetic frame device than for the device with magnetically susceptible frame. The magnetically susceptible frame (which works as

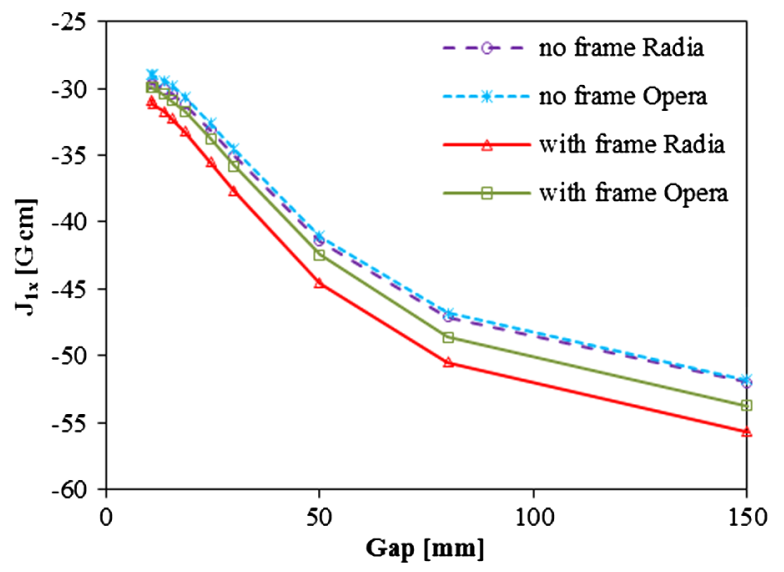

FIG. 7. The horizontal field integrals calculated along the median plane of APS27 \#5s. Comparison of the RADIA and OPERA calculations ( $B_{y}=-0.4, B_{x}=-0.15$ gauss) and frame effect.

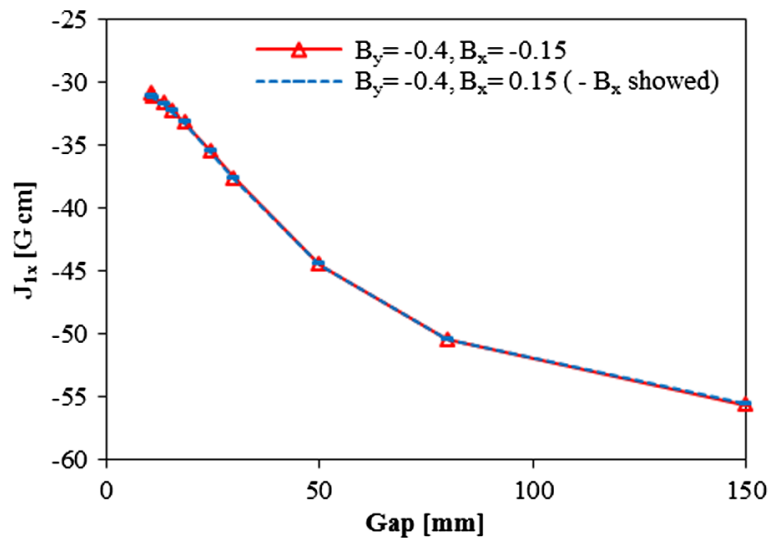

FIG. 8. Comparison of $B_{y}=-0.4, \quad B_{x}=-0.15$ and $B_{y}=-0.4, B_{x}=0.15$ gauss for APS27 \#5s with frame (RADIA calculations).

a shield) compensates for this effect. The enhancement of $J_{1 y E}$ and its gap dependence can be seen in Fig. 11 for cases with a nonmagnetic frame, as compared to a frame that could be magnetized.

\section{Comparison of calculated and measured data}

The differences between measured and calculated (especially for APS27 \#5s) curves in Fig. 12 probably are associated with the method used to calculate the Earth's field contribution. It was assumed that this component is the same for nonrotated devices and devices that have been rotated by $180^{\circ}$. Asymmetry of the device in the $x$ direction, which is very strong for APS27 \#5s, could be the source of the discrepancy. Details of the field distribution along the $z$ axis are shown in Fig. 13. This field distribution was calculated in the middle plane of APS27 \#5s with and without a magnetic frame.

\section{Simulations for an ID with the horizontal main field}

The trajectory straightness of a charged particle passing through the undulator is critical for a free-electron laser (FEL) performance. Because of this, investigation of the

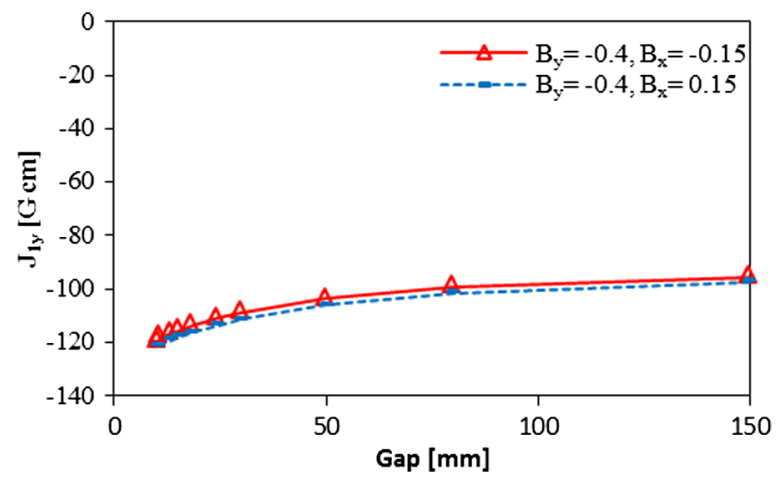

FIG. 9. Comparison $B_{y}=-0.4, B_{x}=-0.15$ and $B_{y}=-0.4$, $B_{x}=0.15$ for APS27 \#5s with frame (RADIA calculations). 


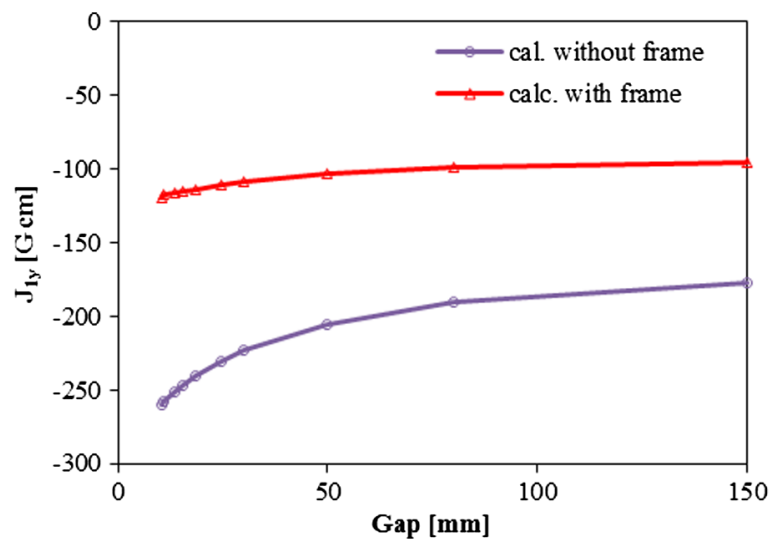

FIG. 10. First vertical field integrals vs gap in case with frame and without it for APS27 \#5s (external field $B_{y}=-0.4$ gauss).

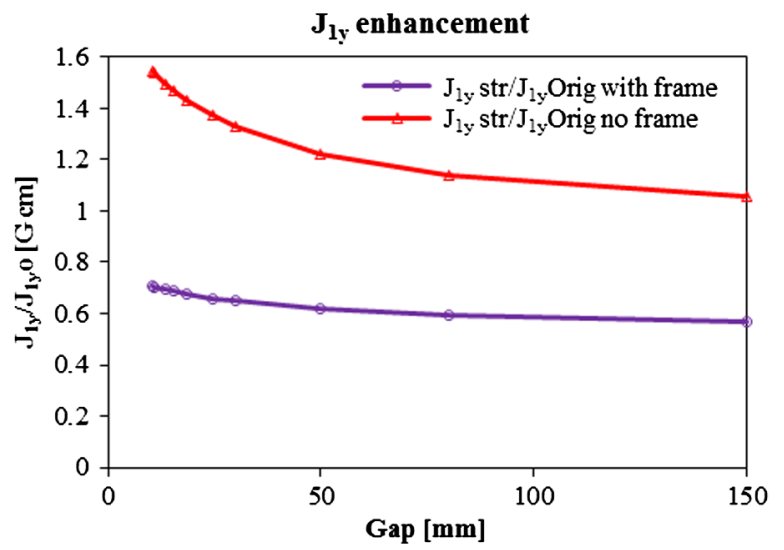

FIG. 11. Vertical Earth's field ( $B_{y}=-0.4$ gauss) enhancement vs gap in the cases with a frame and without it. Calculations are for APS27 \#5s.

Earth field started at the APS during the design of the undulators for LCLS project, when the main requirements were defined [11]. The implementation of $\mu$ shield for the devices with a permanent gap and main vertical filed (horizontally polarizing) helped decrease the propagation of the Earth field by an order of magnitude and solved the problem in this case [6]. A new variable-gap ID with a horizontal main field (vertically polarizing) is currently under construction in the APS as a future prototype for the LCLS II project. Requirements for the field generated by the new device are the same as for the LCLS project. However, implementation of the $\mu$ shields does not appear to be possible; therefore the effect of the Earth field on the new ID should again be considered.

In the case of a vertically polarizing ID (with a horizontal main field), the propagation of the Earth's horizontal field component into the ID's gap is similar to the propagation of the Earth's vertical field component in the horizontally polarizing device (with vertical main field). This is also true for the propagation of the vertical component in the vertically polarizing ID and Earth's horizontal field component in the horizontally polarizing ID. Thus, the vertical Earth's field component is shielded by poles, and the transverse horizontal field is enhanced by them in the case of the ID with a horizontal field. As shown in Fig. 11, the enhancement of the vertical external field is not linear for an ID with a vertical field. Therefore, the situation should be the same for the horizontal component of the Earth's field for an ID with a horizontal field. This makes a horizontal ID (with horizontal main field or vertically polarizing) more sensitive to the change of orientation.

The same calculations discussed above were also performed for the horizontal device. The prototype has a UNA33 magnetic structure (33-mm period) with 48 poles. It is placed on a stand made of magnetic steel. The stand was included in the calculations, which were performed using the OPERA code. The geometry of the simulation model is shown in Fig. 14. Field integrals were calculated for gap range 7-20 mm (Fig. 15). As shown in Fig. 15, the stand shields transverse horizontal external field by a factor of 2. Total $J_{1 x}$ variations compared to the gap are 1.4 and $2.5 \mathrm{G}-\mathrm{cm}$ for the 78.2-cm-long device with the stand and without it, respectively. In a worst-case scenario, if tuning and operational orientations differ by $180^{\circ}$, the variation of
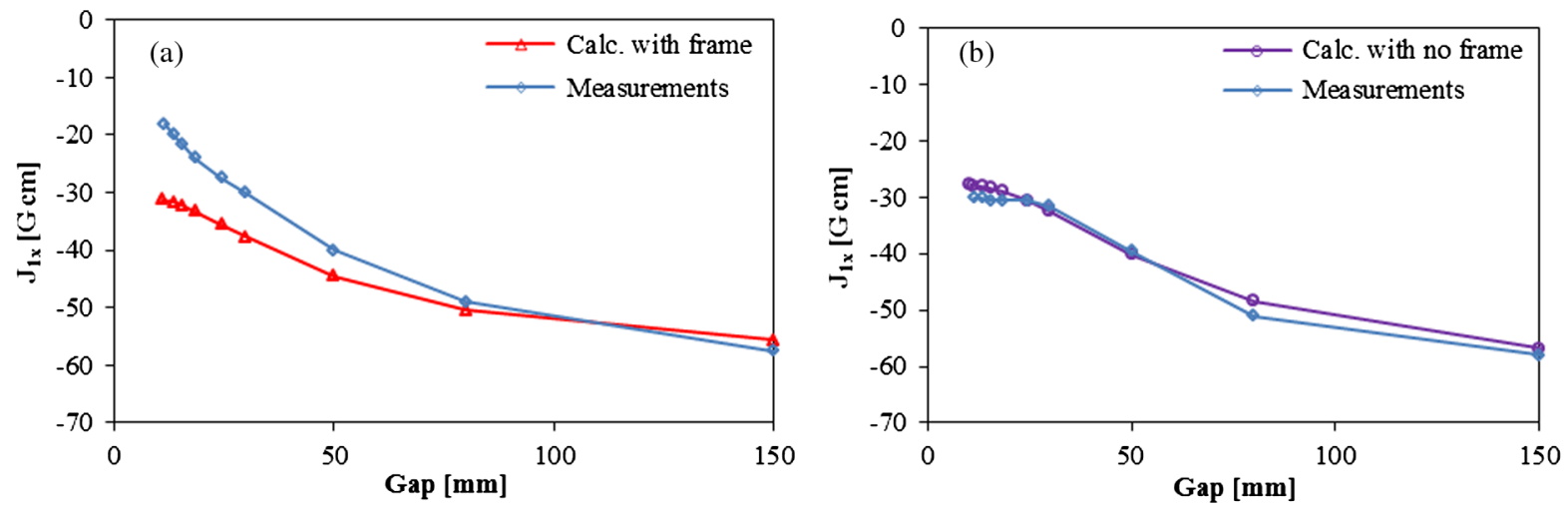

FIG. 12. Transverse horizontal filed integrals obtained from simulations and measurements along the median plane of an ID: (a) APS27 \#5s with magnetic frame; (b) UNA33 \#6. 

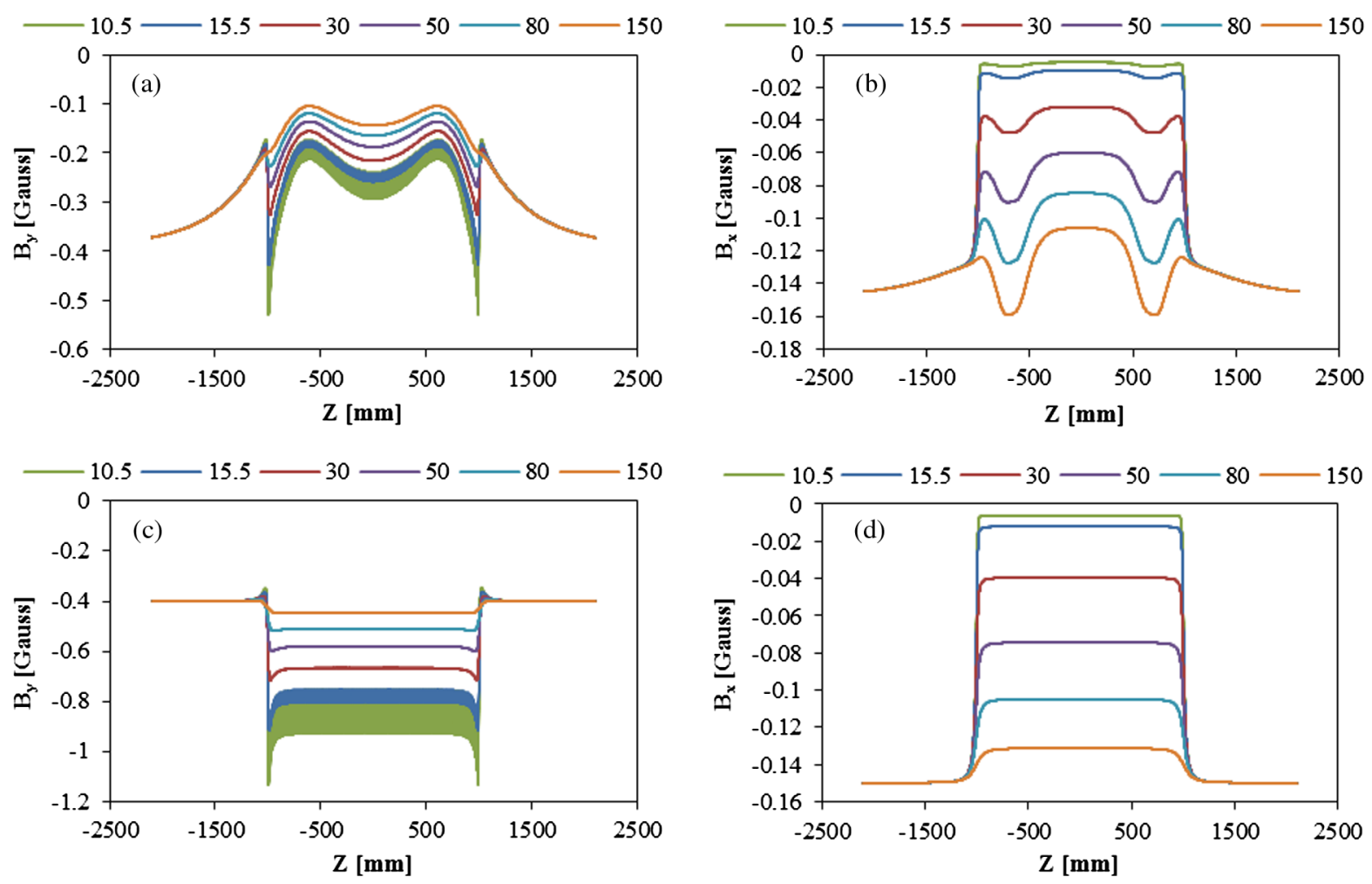

FIG. 13. Earth's field propagation in the APS27 \#5s magnetic structure for different ID's gaps: (a) vertical field propagation with magnetic frame consideration; (b) transverse horizontal field with frame; (c) vertical field without frame; (d) transverse horizontal field without frame.

the first horizontal field integral on the operational position with respect to the magnetic measurement facility will be 2.8 and 5 G-cm for a 78.2-cm-long device or 12 and $22 \mathrm{G}-\mathrm{cm}$ for the full-length undulator segment $(3.4 \mathrm{~m})$ with magnetic and nonmagnetic stand, respectively.

The main requirement for the trajectory is to have the maximum deviation $d$ at the undulator segment $(3.4 \mathrm{~m})$ in both transverse directions within the $2-\mu \mathrm{m}$ range. This requirement stems from the need to minimize the gain length, and consequently the saturation length, for the FEL. The $2-\mu \mathrm{m}$ deviation in both transverse directions gives an increase in gain length of less than $0.2 \%$ [11]. The description of the tolerances and field errors for FEL IDs and their impact on the undulator radiation can be found in Ref. [3]. We assume the Earth field in the ID's gap is homogeneous in order to estimate the additional walk-off caused by the field. Since initial conditions at the entrance to the undulator segment are adjusted to have equal deviations at both segment ends and the middle of the segment (with opposite sign), and since the trajectory has a parabolic profile in a homogeneous field $d=$ $e B_{x, y} L^{2} /\left(16 \gamma m c^{2}\right)$ Thereby, the $2-\mu \mathrm{m}$ walk-off requirement for a 3.4-m-long device transforms to $d=$ $2(0.782 / 3.4)^{2}=0.106 \mu \mathrm{m}$ for a 0.782 -m-long device. Since $J_{2 x, y}(L)=B_{x, y} L^{2} / 2$ for a homogeneous field, the requirement for the second field integrals is $J_{2 x, y} \leq$ $8 d \gamma m c^{2} / e \approx 3.8 \mathrm{kG}-\mathrm{cm}^{2}$ (for $13.5-\mathrm{GeV}$ energy). All

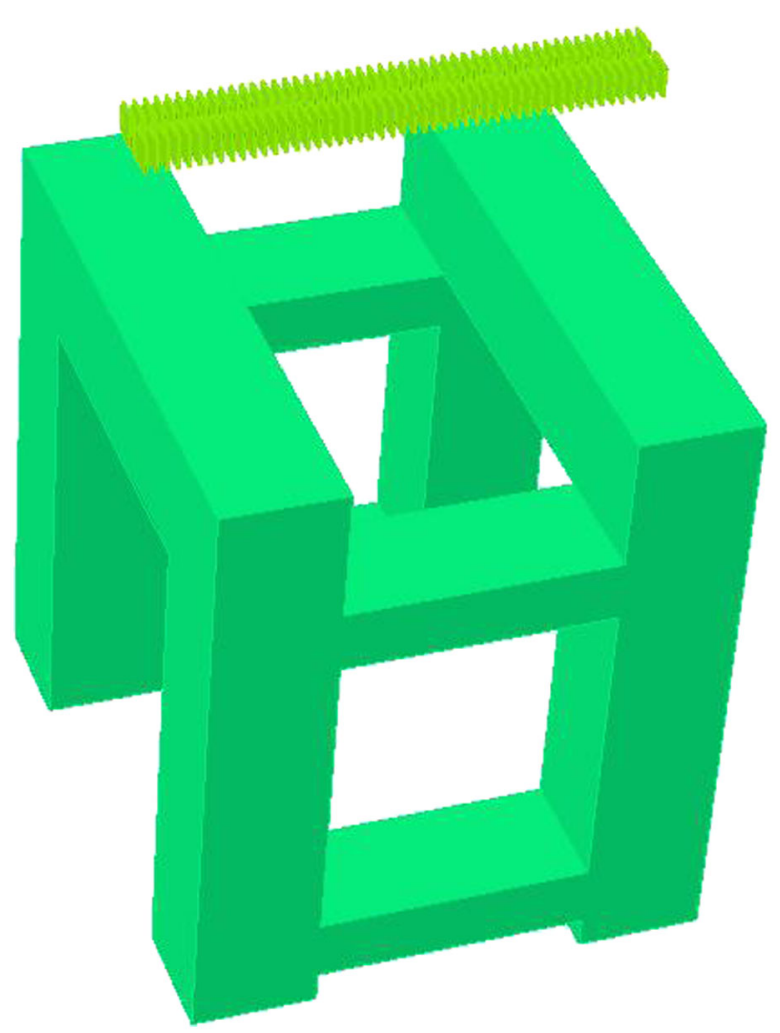

FIG. 14. Simulation model of the horizontal device with stand (UNA33 with 33-mm period magnetic structure). 

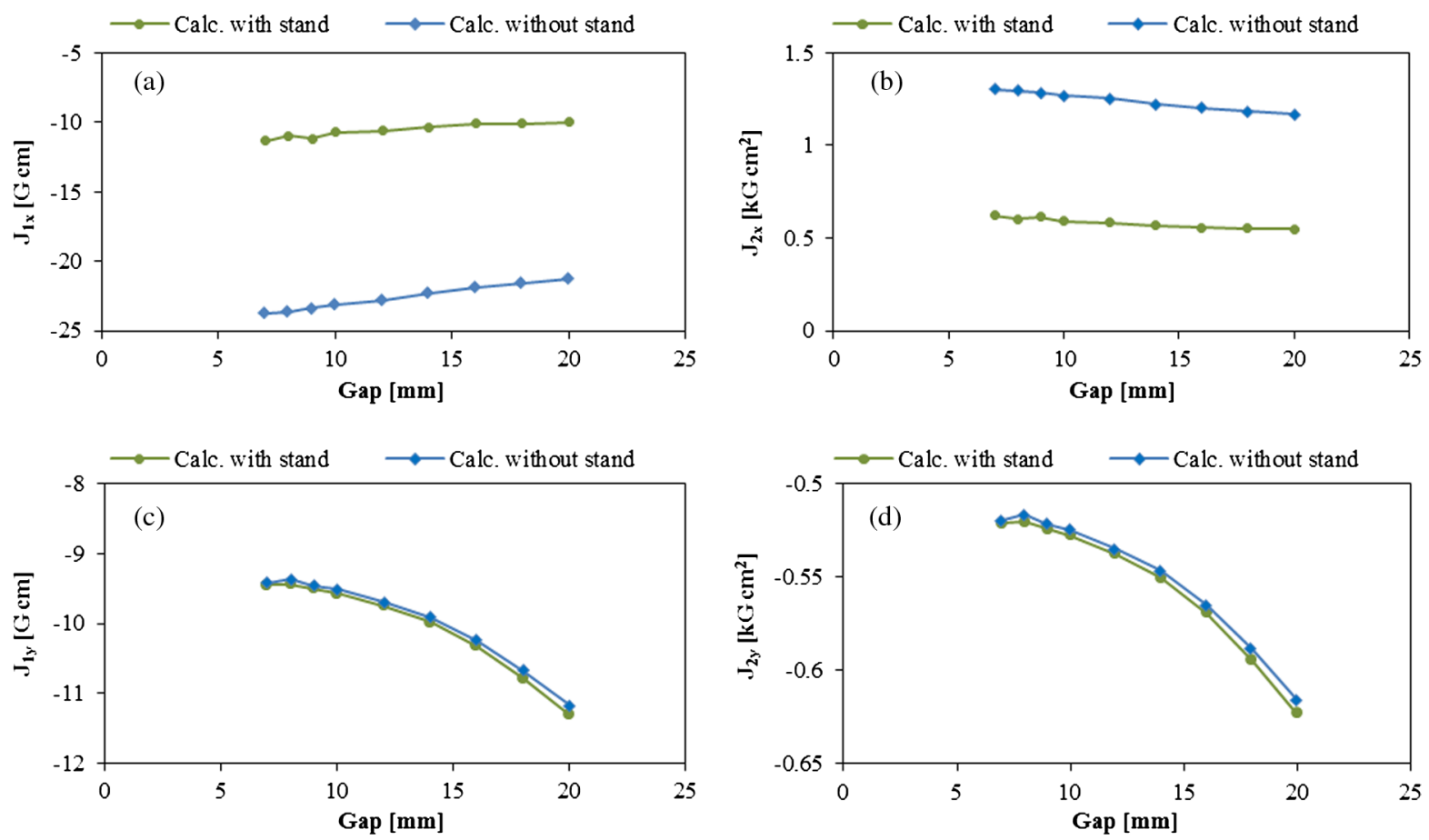

FIG. 15. Field integrals for horizontal undulator (UNA33 with 33-mm period magnetic structure): (a) first horizontal field integral; (b) second horizontal field integral; (c) first vertical field integral; (d) second vertical field integral. External field is $B_{x}=0.15$, $B_{y}=-0.4$ gauss.

calculated second field integrals are less than this value (Fig. 15), and their variation is weak enough within a gap range that they do not disturb particle trajectories significantly.

\section{CONCLUSION}

The magnetic measurements of the two APS IDs APS27 \#5s and UNA33 \#6, with 27- and 33-mm periods, respectively, have been taken in the APS MMF to quantify the influence of the Earth's field on the devices' performance. It was demonstrated that the field integral gap dependences could exceed the specified limits if the effect of the Earth's field is not taken into account for various ID positions on the storage ring.

The effects of the propagation of the Earth's field in the ID gaps as a function of the type of material used for the ID frame, magnetic or nonmagnetic, are very small. Shielding of the horizontal Earth's field component by poles occurs at all gaps. Even at the open gap $(150 \mathrm{~mm})$ the field integral is $-57.5 \mathrm{G}-\mathrm{cm}$, compared to $-63.5 \mathrm{G}-\mathrm{cm}$ measured without devices.

Numerical simulations were done using the RADIA and OPERA codes in order to better interpret experimental results. The simulations were performed for three IDs: APS27 \#5s, UNA33 \#6, and a novel prototype being built for the LCLS II project with a horizontal main field, which is now under development. The enhancement of the vertical/horizontal Earth's field component (in the horizontally/vertically polarizing device) by poles and compensation of this effect by the frame made of magnetically susceptible materials were checked numerically. The calculation showed 0.71 times and 1.55 times enhancement of the vertical Earth's field component for APS27 \#5s at a $10.5-\mathrm{mm}$ gap in the case of a frame with magnetic properties and for the case with no frame, respectively. In addition, the simulations showed that the main LCLS requirement of the $2-\mu \mathrm{m}$ trajectory walk-off per undulator segment $(3.4 \mathrm{~m})$ for the prototype with a horizontal field is not disturbed by the horizontal component of the Earth's field.

\section{ACKNOWLEDGMENTS}

This work is supported by the U.S. Department of Energy, Basic Energy Sciences, Office of Science, under Contract No. DE-AC02-06CH11357. N. O. Strelnikov thanks E. Gluskin for fruitful discussions and advice during the paper's preparation.

\section{APPENDIX A: ID FIELD INTEGRALS}

The field integrals determine the overall effect of the undulator on the electron beam. A trajectory of relativistic electrons moving in an insertion device's (ID's) magnetic field is set by 


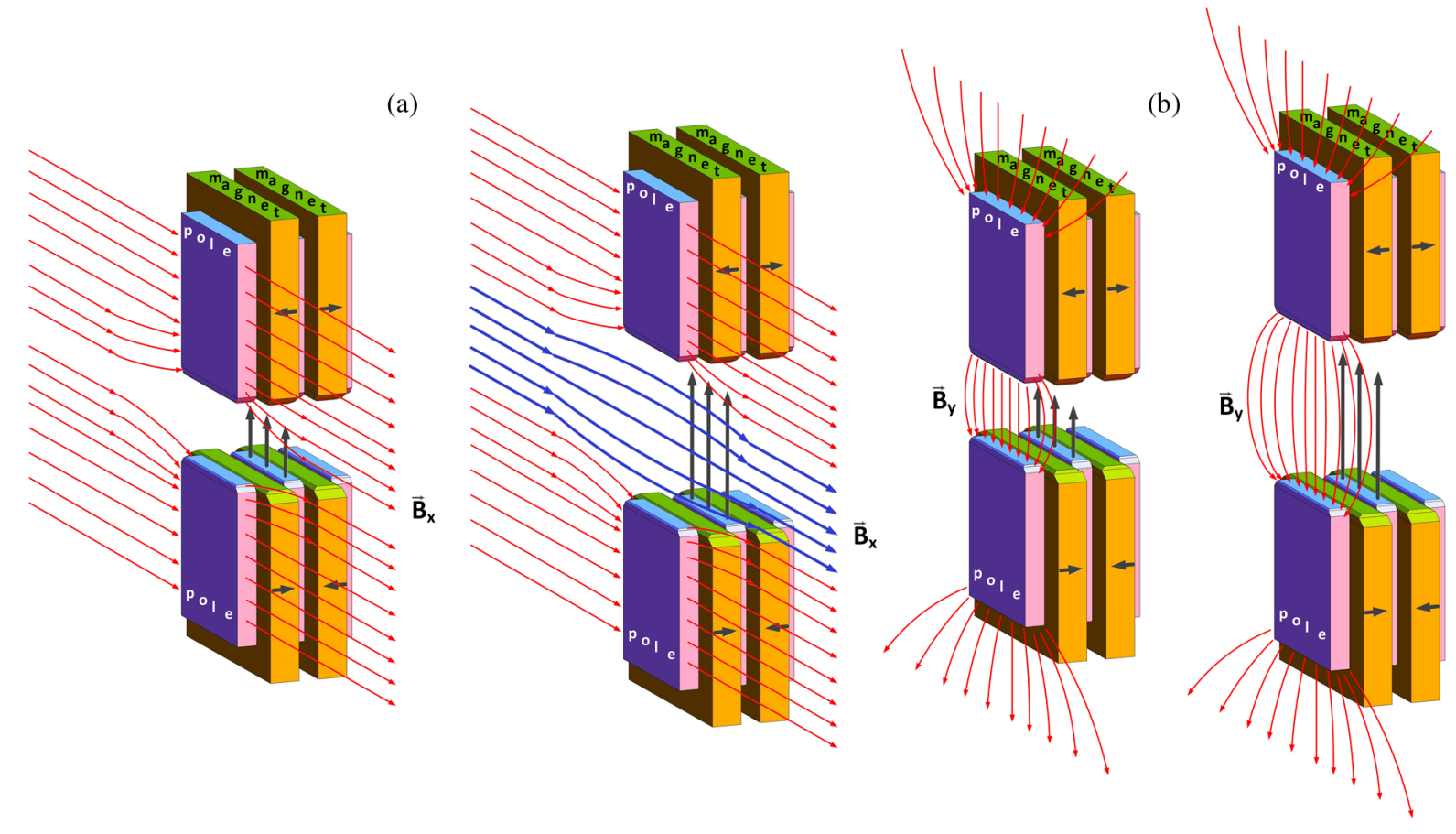

FIG. 16. External field propagation in an ID's gap: (a) horizontal case; (b) vertical case.

$$
\frac{d^{2} x}{d z^{2}} \approx-\frac{e}{\gamma m c^{2}} B_{y}, \quad \frac{d^{2} y}{d z^{2}} \approx \frac{e}{\gamma m c^{2}} B_{x} .
$$

Here $x$ is the horizontal position of an electron transverse to electron motion, $y$ is the vertical position, and $z$ is the position along the longitudinal axis of an ID. Neglecting focusing and the dependence of the undulator field on transverse coordinates $x$ and $y$, these equations have the following solution:

$$
\begin{aligned}
& x^{\prime}=\frac{d x}{d z} \approx-\frac{e}{\gamma m c^{2}} J_{1 y}(z)+x_{0}^{\prime}, \\
& y^{\prime}=\frac{d y}{d z} \approx \frac{e}{\gamma m c^{2}} J_{1 x}(z)+y_{0}^{\prime},
\end{aligned}
$$

where $J_{1 y}(z)=\int_{0}^{z} B_{y}\left(0,0, z_{1}\right) d z_{1}$ is the first vertical field integral, and $J_{1 x}(z)=\int_{0}^{z} B_{x}\left(0,0, z_{1}\right) d z_{1}$ is the first horizontal field integral. Integrating $x^{\prime}$ and $y^{\prime}$, one can get

$$
\begin{aligned}
& x \approx-\frac{e}{\gamma m c^{2}} J_{2 y}(z)+x_{0}^{\prime} z+x_{0}, \\
& y \approx \frac{e}{\gamma m c^{2}} J_{2 x}(z)+y_{0}^{\prime} z+y_{0},
\end{aligned}
$$

where $\quad J_{2 y}(z)=\int_{0}^{z} \int_{0}^{z_{1}} B_{y}\left(0,0, z_{2}\right) d z_{2} d z_{1} \quad$ is the second vertical field integral, and $J_{2 x}(z)=$ $\int_{0}^{z} \int_{0}^{z_{1}} B_{x}\left(0,0, z_{2}\right) d z_{2} d z_{1}$ is the second horizontal field integral. Thus, the first field integrals of an ID define the reference particle angle in the output of the device, and the second field integrals define its offset [3].

\section{APPENDIX B: EXTERNAL FIELD PROPAGATION IN AN ID'S GAP}

In the case of a planar ID with a main vertical field, the penetration of the horizontal component of the Earth's field depends on the ID gap. It is shielded well by the poles at small ID gaps, and to a lesser extent at large gaps. At the same time, a vertical component is enhanced by the poles for such a device (Fig. 16). As is shown in this article, the enhancement of the vertical Earth's field component is weakened by the frame made of magnetically susceptible material.

[1] Advanced Photon Source Upgrade Project, 3-78, 2012, Argonne, IL, USA (Argonne National Laboratory, Lemont, Illinois, 2012), http://www.aps.anl.gov/Upgrade/ FlexPaper_PDR/PDR.pdf.

[2] J. Chavanne et al., in Proceedings of the 2nd International Particle Accelerator Conference, San Sebastian, Spain (EPS-AG, Spain, 2011), THPC153, p. 3245, http://accelconf .web.cern.ch/AccelConf/IPAC2011/papers/thpc153.pdf.

[3] E. Levichev and N. Vinokurov, in Reviews of Accelerator Science and Technology, edited by A. W. Chao and W. Chou (World Scientific Publishing Company, Singapore, 2011), Vol. 3, pp. 203-220.

[4] R. Dejus, I. Vasserman, S. Sasaki, and E. Moog, Report No. ANL/APS/TB-45, 2002, http://www.aps.anl.gov/Science/ Publications/techbulletins/content/files/APS_1421569.pdf.

[5] I. Vasserman and N. Strelnikov, 18th International Magnetic Measurement Workshop, Brookhaven National 
Laboratory (Brookhaven National Laboratory, Upton, New York, 2013).

[6] S. Sasaki and I. Vasserman, in Proceedings of the 27th International Laser Conference, 2005, Stanford, CA, USA (SLAC National Accelerator Laboratory, Menlo Park, California, 2005).

[7] Yong-Chul Chae and G. Decker, in Proceedings of the Particle Accelerator Conference, Dallas, TX, 1995 (IEEE, New York, 1995), Vol. 5, pp. 3409-3411.

[8] P. Elleaume, O. Chubar, and J. Chavanne, in Proceedings of the Particle Accelerator Conference, Vancouver,
BC, Canada, 1997 (IEEE, New York, 1997), pp. 3509-3511.

[9] RADIA 3D magnetostatics computer code, Technical Reference Manual ESRF, Grenoble, France.

[10] OpERA 3D finite element code (TOSCA), Cobham Technical Services, Oxfordshire, England.

[11] E. Gluskin, N. A. Vinokurov, G. Decker, R. J. Dejus, P. Emma, P. Ilinski, E. R. Moog, H.-D. Nuhn, and I. B. Vasserman, Nucl. Instrum. Methods Phys. Res. 475, 323 (2001). 